\title{
Stability and Periodic Nature of A System of Difference Equations
}

\author{
ERKAN TAŞDEMIR
}

Kırklareli University,

Pı narhisar Vocational School of Higher Education, 39300, Kırklareli, Turkey

erkantasdemir@hotmail.com or erkan.tasdemir@klu.edu.tr

In this paper, we investigate the equilibrium points of following a system of difference equations $x_{n+1}=x_{n}{ }_{2} y_{n}-1, y_{n+1}=y_{n}{ }_{2} x_{n}-1$. We also study the asymptotic stability of related system of difference equations. Further we examine the periodic solutions of related system with period two. Additionally, we find out the invariant interval and periodic cycles of related system of difference equations.

2010 AMS Classification: 39A10, 39A30, 39A23

Keywords: Difference equations, equilibrium points, stability, periodicity, invariant

\section{INTRODUCTION}

Difference equations and their systems play a crucial role in different fields of science. Many scientific fields need mathematical models to interpret their results. Especially mathematical models via discrete variables are related to this topic. For the last decades, many scientists have studied stability of equilibrium points, periodicity and boundedness of difference equations or their systems. There are many paper related to difference equations and their systems for examples:

Kent et al, in [8], studied long-term behaviours of solutions of difference equation

$$
x_{n+1}=x_{n} x_{n-1}-1 .
$$

Moreover, in [27], Wang et al and in [16], Liu et al examined convergence of solutions of related difference equation about equilibrium points.

In [10], Kent et al investigated the periodicity of solutions, existence of bounded or unbounded of solutions and stability of solutions of difference equation

$$
x_{n+1}=x_{n-1} x_{n-2}-1 .
$$

Kent et al, in [11], studied the periodicity, stability and unbounded solutions of difference equation

$$
x_{n+1}=x_{n} x_{n-2}-1 .
$$

Further, there are many books and papers related to dynamical systems, see [1] $-[27]$.

In this paper, we investigate the equilibrium points of following a system of difference equations

$$
x_{n+1}=x_{n-2} y_{n}-1, y_{n+1}=y_{n-2} x_{n}-1, n=0,1, \ldots,
$$


where all initial values are real numbers. We also study the asymptotic stability of related system of difference equations. Furthermore, we examine the existence of periodic solutions of related system.

From here to the end of this section, we show useful definitions and theorems which are used during this study.

Firstly, let us introduce discrete dynamical system of the form $(1.2)$

$x_{n+1}=f\left(x_{n}, x_{n-1}, x_{n-2}, y_{n}, y_{n-1}, y_{n-2}\right), y_{n+1}=g\left(x_{n}, x_{n-1}, x_{n-2}, y_{n}, y_{n-1}, y_{n-2}\right)$,

$n=0,1, \ldots$, where $f: I^{3} \times J^{3} \rightarrow I$ and $g: I^{3} \times J^{3} \rightarrow J$ are continuously differentiable functions and $I, J$ are some intervals of real numbers. Moreover, a solution $\left\{\left(x_{n}, y_{n}\right)\right\}_{n=-1}^{\infty}$ of system (1.2) is uniquely determined by initial values $\left(x_{i}, y_{i}\right) \in I \times J$ for $i \in\{-1,0\}$.

Definition 1. Along with the system (1.2), we consider the corresponding vector map $F=\left\{f, x_{n}, x_{n-1}, g, y_{n}, y_{n-1}\right\}$. A point $(\bar{x}, \bar{y})$ is called an equilibrium point of the system (1.2) if

$$
\bar{x}=f(\bar{x}, \bar{x}, \bar{x}, \bar{y}, \bar{y}, \bar{y}), \bar{y}=g(\bar{x}, \bar{x}, \bar{x}, \bar{y}, \bar{y}, \bar{y}) .
$$

The point $(\bar{x}, \bar{y})$ is also called a fixed point of the vector map $F$.

Definition 2. Let $(\bar{x}, \bar{y})$ be an equilibrium point of the system (1.2).

(i): An equilibrium point $(\bar{x}, \bar{y})$ of system (1.2) is called stable if, for every $\varepsilon>0$, there exists $\delta>0$ such that, for every initial value $\left(x_{-i}, y_{-i}\right) \in I \times J$, with

$$
\sum_{i=-1}^{0}\left|x_{i}-\bar{x}\right|<\delta, \quad \sum_{i=-1}^{0}\left|y_{i}-\bar{y}\right|<\delta,
$$

implying $\left|x_{n}-\bar{x}\right|<\varepsilon$ and $\left|y_{n}-\bar{y}\right|<\varepsilon$ for $n \in \mathbb{N}$.

(ii): An equilibrium point $(\bar{x}, \bar{y})$ of system (1.2) is called unstable, if it is not stable.

(iii): An equilibrium point $(\bar{x}, \bar{y})$ of system (1.2) is called locally asymptotically stable if it is stable and if, in addition, there exists $\gamma>0$ such that

$$
\sum_{i=-1}^{0}\left|x_{i}-\bar{x}\right|<\gamma, \quad \sum_{i=-1}^{0}\left|y_{i}-\bar{y}\right|<\gamma,
$$

and $\left(x_{n}, y_{n}\right) \rightarrow(\bar{x}, \bar{y})$ as $n \rightarrow \infty$.

(iv): An equilibrium point $(\bar{x}, \bar{y})$ of system (1.2) is called a global attractor if $\left(x_{n}, y_{n}\right) \rightarrow(\bar{x}, \bar{y})$ as $n \rightarrow \infty$.

(v): An equilibrium point $(\bar{x}, \bar{y})$ of system (1.2) is called globally asymptotically stable if it is stable and a global attractor.

Definition 3. Let $(\bar{x}, \bar{y})$ be an equilibrium point of the map $F$ where $f$ and $g$ are continuously differentiable functions at $(\bar{x}, \bar{y})$. The linearized system of system (1.2) about the equilibrium point $(\bar{x}, \bar{y})$ is

$$
X_{n+1}=F\left(X_{n}\right)=B X_{n}
$$


where

$$
X_{n}=\left(\begin{array}{c}
x_{n} \\
x_{n-1} \\
x_{n-2} \\
y_{n} \\
y_{n-1} \\
y_{n-2}
\end{array}\right)
$$

and $B$ is a Jacobian matrix of system (1.2) about the equilibrium point $(\bar{x}, \bar{y})$.

Definition 4. Assume that $X_{n+1}=F\left(X_{n}\right), n=0,1, \cdots$, is a system of difference equations such that $\bar{X}$ is a fixed point of $F$. If no eigenvalues of the Jacobian matrix $B$ about $\bar{X}$ have absolute value equal to one, then $\bar{X}$ is called hyperbolic. Otherwise, $\bar{X}$ is said to be nonhyperbolic.

Theorem 1 (Linearized Stability Theorem [12], p.11). Assume that

$$
X_{n+1}=F\left(X_{n}\right), n=0,1, \cdots,
$$

is a system of difference equations such that $\bar{X}$ is a fixed point of $F$.

(i): If all eigenvalues of the Jacobian matrix $B$ about $\bar{X}$ lie inside the open unit disk $|\lambda|<1$, that is, if all of them have absolute value less than one, then $\bar{X}$ is locally asymptotically stable.

(ii): If at least one of them has a modulus greater than one, then $\bar{X}$ is unstable.

\section{Equilibrium Points of System (1.1)}

In this here, we examine the equilibrium points of System (1.1).

Theorem 2. System (1.1) has two equilibrium points such that

$$
\begin{aligned}
& \left(\bar{x}_{1}, \bar{y}_{1}\right)=\left(\frac{1-\sqrt{5}}{2}, \frac{1-\sqrt{5}}{2}\right), \\
& \left(\bar{x}_{2}, \bar{y}_{2}\right)=\left(\frac{1+\sqrt{5}}{2}, \frac{1+\sqrt{5}}{2}\right) .
\end{aligned}
$$

Since $\frac{1+\sqrt{5}}{2} \approx 1.618$, the elements of second equilibrium point is equal to the Golden Ratio.

Proof. We can easily seen for the equilibrium points of system (1.1):

$$
\begin{aligned}
& \bar{x}=\bar{x} \cdot \bar{y}-1, \\
& \bar{y}=\bar{y} \cdot \bar{x}-1 .
\end{aligned}
$$

From this system, we obtain

$$
\bar{x}=\bar{x} \cdot \bar{y}-1=\bar{y} .
$$

Thus

$$
\begin{aligned}
\bar{x} & =\bar{x} \cdot \bar{x}-1, \\
\bar{x} & =\bar{x}^{2}-1, \\
\bar{x} & =\frac{1 \pm \sqrt{5}}{2} .
\end{aligned}
$$

So we finished the proof as desired. 


\section{Existence of Periodic and Bounded Solutions of System (1.1)}

In this section, we investigate the periodic behaviours of solutions of System (1.1). Firstly we find out the two periodic solutions of System (1.1). Further, we determine existence of bounded of solutions of System (1.1). Moreover we study the periodic cycles of solutions of System (1.1).

Theorem 3. System (1.1) has periodic solutions with period two.

Proof. Assume that system (1.1) has two periodic solutions. Thus we have for $n \geq 0$ :

$$
x_{2 n}=a, x_{2 n-1}=b, y_{2 n}=c, y_{2 n-1}=d,
$$

where $a \neq b$ and $c \neq d$. Hence we get from system (1.1) and (3.1):

$$
\begin{aligned}
x_{2 n} & =x_{2 n-3} y_{2 n-1}-1 \Rightarrow a=b \cdot d-1, \\
y_{2 n} & =y_{2 n-3} x_{2 n-1}-1 \Rightarrow c=d \cdot b-1, \\
x_{2 n+1} & =x_{2 n-2} y_{2 n}-1 \Rightarrow b=a \cdot c-1, \\
y_{2 n+1} & =y_{2 n-2} x_{2 n}-1 \Rightarrow d=c \cdot a-1 .
\end{aligned}
$$

Therefore we obtain from (3.2)-(3.3) $a=c$ and similarly from (3.4)-(3.5) $b=d$. According to these, we can write the following equations:

$$
\begin{aligned}
& a=b^{2}-1, \\
& b=a^{2}-1 .
\end{aligned}
$$

Now, we write (3.7) into (3.6). Thus we have the following:

$$
a=\left(a^{2}-1\right)^{2}-1 .
$$

When this equation rearrange, we obtain that

$$
a(a+1)\left(a^{2}-a-1\right)=0 .
$$

From this we obtain the four roots of (3.8) as

$$
\begin{aligned}
a & =0, \\
a & =-1, \\
a & =\frac{1-\sqrt{5}}{2}=\bar{x}_{1}=\bar{y}_{1}, \\
a & =\frac{1+\sqrt{5}}{2}=\bar{x}_{2}=\bar{y}_{2} .
\end{aligned}
$$

If $a=0$ or $a=-1$, then System (1.1) has two periodic cycle such as

$$
\left\{\left(x_{n}, y_{n}\right)\right\}=\{\cdots,(-1,-1),(0,0),(-1,-1), \cdots\} .
$$

Since the other values of $a$ are equal to elements of eqilibrium points, they are equilibrium solutions. So, the proof completed as desired.

Now we investigate the invariant interval of System (1.1).

Theorem 4. If the initial values are in $(-1,0)$ then all solutions of System (1.1) are bounded from below and above. 
STABILITy AND PERIOdiC NATURE OF A SYSTEM OF DIFFERENCE EQUATIONS 5

Proof. We assume the initial values of System (1.1) $x_{-2}, x_{-1}, x_{0}, y_{-2}, y_{-1}, y_{0} \in$ $(-1,0)$. Hence we have from System (1.1):

$$
\begin{aligned}
& x_{1}=x_{-2} y_{0}-1 \in(-1,0), \\
& y_{1}=y_{-2} x_{0}-1 \in(-1,0), \\
& x_{2}=x_{-1} y_{1}-1 \in(-1,0), \\
& y_{2}=y_{-1} x_{1}-1 \in(-1,0) .
\end{aligned}
$$

From these and by induction, we obtain that $x_{n}, y_{n} \in(-1,0)$ for all $n \geq 1$.

Remark 1. There are two equations corresponding to the odd and even arguments of $x_{n}$ and $y_{n}$ such that

$$
\begin{aligned}
& u_{n+1}=v_{n-1}\left(u_{n} v_{n-1} u_{n-2}-u_{n}-u_{n-2}\right), \\
& v_{n+1}=u_{n-1}\left(v_{n} u_{n-1} v_{n-2}-v_{n}-v_{n-2}\right) .
\end{aligned}
$$

Firstly we discuss the odd terms of $x_{n}$. Hence we have from System (1.1):

$$
\begin{aligned}
& x_{2 n+3}=x_{2 n} y_{2 n-2}-1 \\
& x_{2 n+3}=\left(x_{2 n-3} y_{2 n-1}-1\right)\left(y_{2 n-1} x_{2 n+1}-1\right)-1 \\
& x_{2 n+3}=y_{2 n-1}\left(x_{2 n-3} y_{2 n-1} x_{2 n+1}-x_{2 n+1}-x_{2 n-3}\right) .
\end{aligned}
$$

Similarly we can write the even terms of $x_{n}$ and the odd and even terms of $y_{n}$ as follows:

$$
\begin{aligned}
x_{2 n+4} & =y_{2 n}\left(x_{2 n-2} y_{2 n} x_{2 n+2}-x_{2 n+2}-x_{2 n-2}\right), \\
y_{2 n+3} & =x_{2 n-1}\left(y_{2 n-3} x_{2 n-1} y_{2 n+1}-y_{2 n+1}-y_{2 n-3}\right) \\
y_{2 n+4} & =x_{2 n}\left(y_{2 n-2} x_{2 n} y_{2 n+2}-y_{2 n+2}-y_{2 n-2}\right) .
\end{aligned}
$$

Therefore $x_{2 n+1}$ and $x_{2 n+2}$ satisfy the following equation

$$
u_{n+1}=v_{n-1}\left(u_{n} v_{n-1} u_{n-2}-u_{n}-u_{n-2}\right)
$$

where $n \in \mathbb{N}$ and $u_{n}, v_{n} \in(-1,0)$.

Likewise $y_{2 n+1}$ and $y_{2 n+2}$ satisfy the equation

$$
v_{n+1}=u_{n-1}\left(v_{n} u_{n-1} v_{n-2}-v_{n}-v_{n-2}\right)
$$

where $n \in \mathbb{N}$ and $u_{n}, v_{n} \in(-1,0)$.

From (3.13) and (3.14), we obtain the following eight equilibrium points:

$$
\begin{aligned}
& \bar{u}_{1}=\bar{v}_{1}=-1, \\
& \bar{u}_{2}=\bar{v}_{2}=0, \\
& \bar{u}_{3}=\bar{v}_{3}=\frac{1-\sqrt{5}}{2}, \\
& \bar{u}_{4}=\bar{v}_{4}=\frac{1+\sqrt{5}}{2} .
\end{aligned}
$$

Now we take the $f$ and $g$ functions corresponding to (3.13) and (3.14) respectively:

$$
f(u, v, w)=g(u, v, w)=w(u w v-u-v) .
$$

where $u, v, w \in(-1,0)$. Then we obtain the followings:

$$
\begin{aligned}
f_{u} & =g_{u}=w^{2} v-w>0 \\
f_{v} & =g_{v}=w^{2} u-w>0 \\
f_{w} & =g_{w}=2 u v w-u-v>0 .
\end{aligned}
$$


So, the $f$ and $g$ functions are strictly increasing in each argument.

Theorem 5. Let the initial values be in $(-1,0)$.

(H1): $x_{-2}<\bar{x}_{1}, x_{-1}>\bar{x}_{1}, x_{0}<\bar{x}_{1}, y_{-2}<\bar{y}_{1}, y_{-1}>\bar{y}_{1}$ and $y_{0}<\bar{y}_{1}$.

(H2): $x_{-2}>\bar{x}_{1}, x_{-1}<\bar{x}_{1}, x_{0}>\bar{x}_{1}, y_{-2}>\bar{y}_{1}, y_{-1}<\bar{y}_{1}$ and $y_{0}>\bar{y}_{1}$.

(a): If (H1) holds then there is an $N \in \mathbb{N}_{0}$ such that $x_{2 n+1}>\bar{x}_{1}, x_{2 n+2}<\bar{x}_{1}$, $y_{2 n+1}>\bar{y}_{1}$ and $y_{2 n+2}<\bar{y}_{1}$.

(b): If (H2) holds then there is an $N \in \mathbb{N}_{0}$ such that $x_{2 n+1}<\bar{x}_{1}, x_{2 n+2}>\bar{x}_{1}$, $y_{2 n+1}<\bar{y}_{1}$ and $y_{2 n+2}>\bar{y}_{1}$.

Proof. According to Theorem 4, if the initial values $x_{-2}, x_{-1}, x_{0}, y_{-2}, y_{-1}, y_{0} \in$ $(-1,0)$ then we have $x_{n}, y_{n} \in(-1,0)$ for all $n \geq 1$. As the proof of (b) is similar to (a), we will prove only (a). From (H1), we have $-1<x_{-2}, x_{0}, y_{-2}, y_{0}<\bar{x}_{1}=\bar{y}_{1}$. So we obtain that

$$
\begin{aligned}
& x_{1}=x_{-2} y_{0}-1>\bar{x}_{1}^{2}-1=\bar{x}_{1}, \\
& y_{1}=y_{-2} x_{0}-1>\bar{y}_{1}^{2}-1=\bar{y}_{1} .
\end{aligned}
$$

Hence we have that $\bar{x}_{1}<x_{1}<0, \bar{y}_{1}<y_{1}<0$. From (H1), we get $\bar{x}_{1}<x_{-1}<0$, $\bar{y}_{1}<y_{-1}<0$. Therefore we obtain the followings:

$$
\begin{aligned}
& x_{2}=x_{-1} y_{1}-1<\bar{x}_{1}^{2}-1=\bar{x}_{1}, \\
& y_{2}=y_{-1} x_{1}-1<\bar{y}_{1}^{2}-1=\bar{y}_{1} .
\end{aligned}
$$

So, by induction we obtain that

$$
\begin{aligned}
x_{2 k+3} & =x_{2 k} y_{2 k+2}-1>\bar{x}_{1}^{2}-1=\bar{x}_{1}, \\
x_{2 k+4} & =x_{2 k+1} y_{2 k+3}-1<\bar{x}_{1}^{2}-1=\bar{x}_{1}, \\
y_{2 k+3} & =y_{2 k} x_{2 k+2}-1>\bar{y}_{1}^{2}-1=\bar{y}_{1}, \\
y_{2 k+4} & =y_{2 k+1} x_{2 k+3}-1<\bar{y}_{1}^{2}-1=\bar{y}_{1} .
\end{aligned}
$$

Therefore the proof completed.

Theorem 6. Suppose that $x_{-2}, x_{-1}, x_{0}, y_{-2}, y_{-1}, y_{0} \in(-1,0)$ and (H1) or (H2) hold. Then both $\left\{x_{n}\right\}_{n=-2}^{\infty}$ and $\left\{y_{n}\right\}_{n=-2}^{\infty}$ converge to a two-cycle $\{-1,0\}$.

Proof. Firstly, from Theorem 4, we know that $x_{n}, y_{n} \in(-1,0)$, for $n \geq 1$.

(a): If for some $N \geq-1, u_{n}>\bar{u}_{3}$ for $n \geq N$ then $\left(u_{n}\right)_{n=-1}^{\infty}$ of (3.13) converges to $\bar{u}_{2}=0$ where $u_{n} \in(-1,0)=\left(\bar{u}_{1}, \bar{u}_{2}\right)$ for $n \geq-1$.

(b): If for some $N \geq-1, u_{n}<\bar{u}_{3}$ for $n \geq N$ then $\left(u_{n}\right)_{n=-1}^{\infty}$ of (3.13) converges to $\bar{u}_{1}=-1$ where $u_{n} \in(-1,0)=\left(\bar{u}_{1}, \bar{u}_{2}\right)$ for $n \geq-1$.

(c): If for some $N \geq-1, v_{n}>\bar{v}_{3}$ for $n \geq N$ then $\left(v_{n}\right)_{n=-1}^{\infty}$ of (3.14) converges to $\bar{v}_{2}=0$ where $v_{n} \in(-1,0)=\left(\bar{v}_{1}, \bar{v}_{2}\right)$ for $n \geq-1$.

(d): If for some $N \geq-1, v_{n}<\bar{v}_{3}$ for $n \geq N$ then $\left(v_{n}\right)_{n=-1}^{\infty}$ of (3.14) converges to $\bar{v}_{2}=-1$ where $v_{n} \in(-1,0)=\left(\bar{v}_{1}, \bar{v}_{2}\right)$ for $n \geq-1$.

Now we will prove (a). The proofs of (b), (c) and (d) are similar to (a), so we leave it to readers.

We know from Theorem 5:

$$
u_{n} \in\left(\bar{u}_{3}, \bar{u}_{2}\right), n \geq N .
$$

Let $I=\lim _{n \rightarrow \infty} \inf u_{n}$ and $S=\lim _{n \rightarrow \infty} \sup u_{n}$. Hence we get $\bar{u}_{3} \leq I \leq S \leq \bar{u}_{2}=0$. 
We assume that $I=\bar{u}_{3}$. From (3.15), there is an $\varepsilon>0$ such that

$$
I+\varepsilon<u_{N}, u_{N+1}, u_{N+2}<\bar{u}_{2} .
$$

Since $f$ is a monotonic function, we can write

$$
f(x, x, x)>x \text { for } x \in\left(\bar{u}_{3}, \bar{u}_{2}\right) .
$$

Hence we get

$$
u_{N+3}=f\left(u_{N+2}, u_{N+1}, u_{N}\right)>f(I+\varepsilon, I+\varepsilon, I+\varepsilon)>I+\varepsilon .
$$

Therefore we obtain by induction $u_{n}>I+\varepsilon, n \geq N$. And so $\lim _{n \rightarrow \infty} \inf u_{n} \geq I+\varepsilon$. It is a contradiction.

We assume that $I \in\left(\bar{u}_{3}, \bar{u}_{2}\right)$. Let $\left(u_{n_{k}}\right)_{k \in \mathbb{N}}$ be a subsequence of $\left(u_{n}\right)_{n=-1}^{\infty}$ such that $\lim _{k \rightarrow \infty} u_{n_{k}}=I$. Thus we can denote the subsequences such that there are limits: $\lim _{k \rightarrow \infty} u_{n_{k}-1}=K_{-1}, \lim _{k \rightarrow \infty} u_{n_{k}-2}=K_{-2}$ and $\lim _{k \rightarrow \infty} u_{n_{k}-3}=K_{-3}$. From (3.16), we have

$$
f\left(K_{-1}, K_{-2}, K_{-3}\right)=I<f(I, I, I) .
$$

Hence there is an $i_{0} \in\{1,2,3\}$ such that $K_{-i_{0}}<I$. Otherwise, $K_{-i_{0}} \geq I$ for $i=1,2,3$ and monotonicity of $f$ we have

$$
f(I, I, I) \leq f\left(K_{-1}, K_{-2}, K_{-3}\right)=I<f(I, I, I) .
$$

This is a contradiction. On the other hand, if $K_{-i_{0}}<I$ then it contradicts the choice of $I$. So $I$ cannot be in $\left(\bar{u}_{3}, \bar{u}_{2}\right)$. Therefore we obtain $\bar{u}_{2} \leq I \leq S \leq \bar{u}_{2}$. Hence $\lim _{n \rightarrow \infty} u_{n}=\bar{u}_{2}=0$. The proof of (a) is completed.

Example 1. Consider the System (1.1) with the initial values $x_{-2}=-0.9, x_{-1}=$ $-0.2, x_{0}=-0.7, y_{-2}=-0.8, y_{-1}=-0.3$ and $y_{0}=-0.9$. Then System (1.1) is bounded from below and above. Moreover, the solutions of System (1.1) converges to a two periodic cycle $\{-1,0\}$ such as Theorem 6 . We can easily see the followings:

$$
\lim _{n \rightarrow \infty} x_{2 n}=-1, \lim _{n \rightarrow \infty} x_{2 n+1}=0, \lim _{n \rightarrow \infty} y_{2 n}=-1, \lim _{n \rightarrow \infty} y_{2 n+1}=0 .
$$

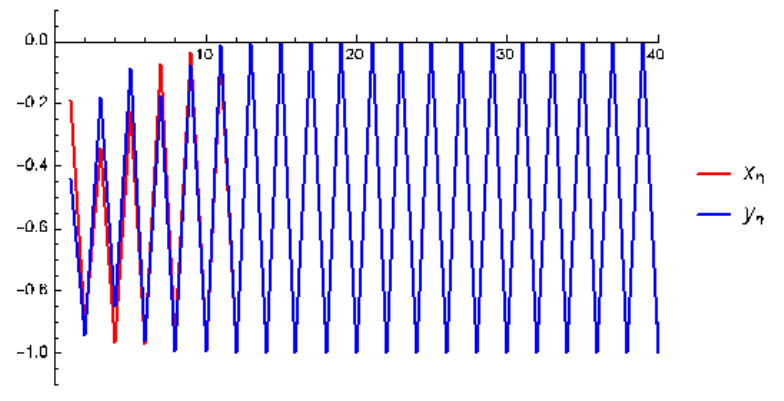

Figure 1. The plot of System (1.1) with $x_{-2}=-0.9, x_{-1}=-0.2$, $x_{0}=-0.7, y_{-2}=-0.8, y_{-1}=-0.3$ and $y_{0}=-0.9$. 


\section{Stability Analysis of System (1.1)}

This section, we study the stability of System (1.1). Moreover, we determine that both negative and positive equilibrium points of System (1.1) are unstable.

Theorem 7. Equilibrium point $\left(\bar{x}_{1}, \bar{y}_{1}\right)$ of System (1.1) is locally unstable.

Proof. Firstly we study linearized form of System (1.1). For this, we consider the transformation:

$$
\left(x_{n}, x_{n-1}, x_{n-2}, y_{n}, y_{n-1}, y_{n-2}\right) \rightarrow\left(f, f_{1}, f_{2}, g, g_{1}, g_{2}\right),
$$

where

$$
\begin{aligned}
f & =x_{n-2} y_{n}-1, \\
f_{1} & =x_{n}, \\
f_{2} & =x_{n-1}, \\
g & =y_{n-2} x_{n}-1, \\
g_{1} & =y_{n}, \\
g_{2} & =y_{n-1} .
\end{aligned}
$$

Therefore we have the Jacobian matrix about equilibrium point $(\bar{x}, \bar{y})$ :

$$
B(\bar{x}, \bar{y})=\left(\begin{array}{cccccc}
0 & 0 & \bar{y} & \bar{x} & 0 & 0 \\
1 & 0 & 0 & 0 & 0 & 0 \\
0 & 1 & 0 & 0 & 0 & 0 \\
\bar{y} & 0 & 0 & 0 & 0 & \bar{x} \\
0 & 0 & 0 & 1 & 0 & 0 \\
0 & 0 & 0 & 0 & 1 & 0
\end{array}\right) .
$$

Thus, the linearized system about the equilibrium point $(\bar{x}, \bar{y})=\left(\frac{1-\sqrt{5}}{2}, \frac{1-\sqrt{5}}{2}\right)$ is $X_{N+1}=B(\bar{x}, \bar{y}) X_{n}$ where $X_{n}=\left(\left(x_{n}, x_{n-1}, x_{n-2}, y_{n}, y_{n-1}, y_{n-2}\right)\right)^{T}$ and

$$
B(\bar{x}, \bar{y})=\left(\begin{array}{cccccc}
0 & 0 & \frac{1-\sqrt{5}}{2} & \frac{1-\sqrt{5}}{2} & 0 & 0 \\
1 & 0 & 0 & 0 & 0 & 0 \\
0 & 1 & 0 & 0 & 0 & 0 \\
\frac{1-\sqrt{5}}{2} & 0 & 0 & 0 & 0 & \frac{1-\sqrt{5}}{2} \\
0 & 0 & 0 & 1 & 0 & 0 \\
0 & 0 & 0 & 0 & 1 & 0
\end{array}\right)
$$

So, the characteristic equation of $B(\bar{x}, \bar{y})$ is

$$
\lambda^{6}+\left(\frac{\sqrt{5}-3}{2}\right) \lambda^{4}+(\sqrt{5}-1) \lambda^{3}+\frac{3-\sqrt{5}}{2}=0 .
$$

Hence, we have six roots of Eq.(4.1):

$$
\begin{gathered}
\left|\lambda_{1}\right|=|-1.11508|=1.11508 \\
\left|\lambda_{2,3}\right|=|0.248524 \pm 0.701773 i|=0.74448 \\
\left|\lambda_{4,5}\right|=|0.65298 \pm 0.687037 i|=0.94784 \\
\left|\lambda_{6}\right|=|-0.687925|=0.687925
\end{gathered}
$$

Due to

$$
\left|\lambda_{1}\right|>1>\left|\lambda_{4,5}\right|>\left|\lambda_{2,3}\right|>\left|\lambda_{6}\right|
$$


STABILITY AND PERIODIC NATURE OF A SYSTEM OF DIFFERENCE EQUATIONS 9

and from linearized stability theorem, five roots of the characteristic equation lie inside the unit disk but the other root lie outside the unit disk. So, the negative equilibrium of System (1.1) is locally unstable.

Theorem 8. Equilibrium point $\left(\bar{x}_{2}, \bar{y}_{2}\right)$ of System (1.1) is locally unstable.

Proof. Firstly we study linearized form of System (1.1). For this, we consider the transformation:

$$
\left(x_{n}, x_{n-1}, x_{n-2}, y_{n}, y_{n-1}, y_{n-2}\right) \rightarrow\left(f, f_{1}, f_{2}, g, g_{1}, g_{2}\right),
$$

where

$$
\begin{aligned}
f & =x_{n-2} y_{n}-1, \\
f_{1} & =x_{n}, \\
f_{2} & =x_{n-1}, \\
g & =y_{n-2} x_{n}-1, \\
g_{1} & =y_{n}, \\
g_{2} & =y_{n-1} .
\end{aligned}
$$

Therefore we have the Jacobian matrix about equilibrium point $(\bar{x}, \bar{y})$ :

$$
B(\bar{x}, \bar{y})=\left(\begin{array}{cccccc}
0 & 0 & \bar{y} & \bar{x} & 0 & 0 \\
1 & 0 & 0 & 0 & 0 & 0 \\
0 & 1 & 0 & 0 & 0 & 0 \\
\bar{y} & 0 & 0 & 0 & 0 & \bar{x} \\
0 & 0 & 0 & 1 & 0 & 0 \\
0 & 0 & 0 & 0 & 1 & 0
\end{array}\right)
$$

Thus, the linearized system about the equilibrium point $(\bar{x}, \bar{y})=\left(\frac{1+\sqrt{5}}{2}, \frac{1+\sqrt{5}}{2}\right)$ is $X_{N+1}=B(\bar{x}, \bar{y}) X_{n}$ where $X_{n}=\left(\left(x_{n}, x_{n-1}, x_{n-2}, y_{n}, y_{n-1}, y_{n-2}\right)\right)^{T}$ and

$$
B(\bar{x}, \bar{y})=\left(\begin{array}{cccccc}
0 & 0 & \frac{1+\sqrt{5}}{2} & \frac{1+\sqrt{5}}{2} & 0 & 0 \\
1 & 0 & 0 & 0 & 0 & 0 \\
0 & 1 & 0 & 0 & 0 & 0 \\
\frac{1+\sqrt{5}}{2} & 0 & 0 & 0 & 0 & \frac{1+\sqrt{5}}{2} \\
0 & 0 & 0 & 1 & 0 & 0 \\
0 & 0 & 0 & 0 & 1 & 0
\end{array}\right)
$$

So, the characteristic equation of $B(\bar{x}, \bar{y})$ is

$$
\lambda^{6}-\left(\frac{\sqrt{5}+3}{2}\right) \lambda^{4}-(1+\sqrt{5}) \lambda^{3}+\frac{3+\sqrt{5}}{2}=0 .
$$

Hence, we have four roots of Eq.(4.2):

$$
\begin{aligned}
\left|\lambda_{1}\right| & =\left|\lambda_{2}\right|=|-1.21673+0.709835 i|=1.4087 \\
\left|\lambda_{3}\right| & =\left|\lambda_{4}\right|=|-0.199036+0.873464 i|=0.89585 \\
\left|\lambda_{5}\right| & =|0.815421|=0.815421 \\
\left|\lambda_{6}\right| & =|2.01611|=2.01611
\end{aligned}
$$

Because of

$$
\left|\lambda_{5}\right|<\left|\lambda_{3}\right|=\left|\lambda_{4}\right|<1<\left|\lambda_{1}\right|=\left|\lambda_{2}\right|<\left|\lambda_{6}\right|,
$$


and from linearized stability theorem, three roots of the characteristic equation lie inside the unit disk but the other roots lie outside the unit disk. So, the positive equilibrium of System (1.1) is locally unstable.

\section{Conclusion}

In this study, we determine the equilibrium points of System (1.1). We also examine the periodicity of solutions of System (1.1) with period two. Moreover we present that if the initial values of System $(1.1)$ are in $(-1,0)$ then solutions of System (1.1) are both bounded and converge to a two periodic cycle $\{-1,0\}$.

\section{REFERENCES}

[1] Agarwal RP, Wong PJ. Advanced topics in difference equations (Vol. 404). Springer Science \& Business Media, 2013.

[2] Camouzis E, Ladas G. Dynamics of third order rational difference equations with open problems and conjectures, volume 5 of Advances in Discrete Mathematics and Applications, Chapman \& Hall/CRC, Boca Raton, FL; 2008.

[3] Din Q, Qureshi MN, Khan AQ. Dynamics of a fourth-order system of rational difference equations. Adv. Difference Equ. 2012; 2012:215, pp. 1-15.

[4] Elaydi S. An Introduction to Difference Equations. Springer-Verlag, 1996.

[5] Gelisken A, Kara M. Some general systems of rational difference equations. Journal of Difference Equations 2015; 2015, pp. 1-7.

[6] Göcen M, Cebeci A. On the Periodic Solutions of Some Systems of Higher Order Difference Equations. Rocky Mountain J. Math. 2018; 48 (3), pp. 845-858.

[7] Grove EA, Ladas G. Periodicities in nonlinear difference equations. Chapman and Hall/CRC, 2004.

[8] Kent CM, Kosmala W, Radin MA, Stevic S. Solutions of the difference equation $x_{n+1}=$ $x_{n} x_{n-1}-1$. Abstr. Appl. Anal. 2010; 2010, pp. 1-13.

[9] Kent CM, Kosmala W. On the Nature of Solutions of the Difference Equation $x_{n+1}=$ $x_{n} x_{n-3}-1$. International Journal of Nonlinear Analysis and Applications 2011; 2 (2), pp. $24-43$.

[10] Kent CM, Kosmala W, Stević S. Long-term behavior of solutions of the difference equation $x_{n+1}=x_{n-1} x_{n-2}-1$. Abstr. Appl. Anal. 2010; 2010, pp. 1-17.

[11] Kent CM, Kosmala W, Stević S. On the difference equation $x_{n+1}=x_{n} x_{n-2}-1$. Abstr. Appl. Anal. 2011; 2011, pp. 1-15.

[12] Kocic VL, Ladas G. Global behavior of nonlinear difference equations of higher order with applications. Vol. 256. Springer Science \& Business Media, 1993.

[13] Kulenovic MR, Ladas G. Dynamics of second order rational difference equations: with open problems and conjectures. Chapman and Hall/CRC, 2001.

[14] Kulenovic MR, Nurkanovic M. Asymptotic behavior of a system of linear fractional difference equations. J. Inequal. Appl. 2005; 2005 (2), pp. 1-17.

[15] Kurbanlı AS, Çinar C, Yalçinkaya İ. On the behavior of positive solutions of the system of rational difference equations $\mathrm{xn}+1=\mathrm{xn}-1 \mathrm{ynxn}-1+1$, yn $+1=\mathrm{yn}-1 \mathrm{xnyn}-1+1$. Mathematical and Computer Modelling 2011; 53 (5-6), pp. 1261-1267.

[16] Liu K, Li P, Han F, Zhong W. Behavior of the Difference Equations x n+ 1= x n x n-1-1. J. Comput. Anal. Appl. 2017; 201722 (7), pp. 1361-1370.

[17] Okumuş I, Soykan Y. Some Technique To Show The Boundedness Of Rational Difference Equations. Journal of Progressive Research in Mathematics 2018; 13 (2), pp. 2246-2258.

[18] Okumuş İ, Soykan Y. Dynamical behavior of a system of three-dimensional nonlinear difference equations. Adv. Difference Equ. 2018; 2018:224, pp. 1-15.

[19] Stevic S, Alghamdi MA, Maturi DA, Shahzad N. On the Periodicity of Some Classes of Systems of Nonlinear Difference Equations. Abstr. Appl. Anal. 2014; 2014, pp. 1-6.

[20] Stevic S. On the difference equation $x_{n+1}=\alpha+x_{n-1} / x_{n}$. Comput. Math. Appl. 2008; 56, pp. 1159-1171.

[21] Taşdemir E. On the Asymptotically Periodic Solutions of A Fifth Order Difference Equation. J. Math. Anal. 2019; 10 (3), pp. 100-111. 
[22] Taşdemir E. On The Dynamics of a Nonlinear Difference Equation. Adiyaman University Journal of Science 2019; 9 (1), pp. 190-201.

[23] Taşdemir E, Soykan Y. On the Periodicies of the Difference Equation $x_{n+1}=x_{n} x_{n-1}+\alpha$. Karaelmas Science and Engineering Journal 2016; 6 (2), pp. 329-333.

[24] Taşdemir E, Soykan Y. Long-Term Behavior of Solutions of the Non-Linear Difference Equation $x_{n+1}=x_{n-1} x_{n-3}-1$. Gen. Math. Notes 2017; 38 (1), pp. 13-31.

[25] Taşdemir E, Soykan Y. Stability of Negative Equilibrium of a Non-Linear Difference Equation. J. Math. Sci. Adv. Appl. 2018; 49 (1), pp. 51-57.

[26] Taşdemir E, Soykan Y. Dynamical Analysis of a Non-Linear Difference Equation. J. Comput. Anal. Appl. 2019; 26 (2), pp. 288-301.

[27] Wang Y, Luo Y, Lu Z. Convergence of solutions of $x_{n+1}=x_{n} x_{n-1}-1$. Appl. Math. E-Notes $2012 ; 12$, pp. 153-157. 